\title{
Impact of sphingomyelin levels on coronary heart disease and left ventricular systolic function in humans
}

\author{
Xueying Chen ${ }^{1+}$, Aijun Sun ${ }^{1+}$, Yunzeng Zou ${ }^{1}$, Junbo Ge ${ }^{1 *}$, Jason M Lazar ${ }^{2 *}$ and Xian-Cheng Jiang ${ }^{3}$
}

\begin{abstract}
Sphingomyelin (SM) is an abundant phospholipid in cell membranes and in lipoproteins. In human plasma, SM is mainly found in atherogenic lipoproteins; therefore, higher levels of SM may promote atherogenesis. We investigated the relations between plasma SM levels and the presence of angiographic coronary heart disease (CHD) and left ventricular systolic dysfunction. We studied 732 patients referred for coronary angiography. Median SM levels were higher among patients with CHD and in those with LV systolic dysfunction (LVEF $<50 \%$ ) than in patients without CHD or LV dysfunction. SM levels were significantly correlated with fibrinogen levels, diabetes, $\mathrm{apoB}$, and triglyceride levels. On multivariate analyses, higher median SM levels were associated with a higher risk of $\mathrm{CHD}$ and lower LV ejection fraction. The pro-atherogenic property of plasma SM might be related to 1) CHD; 2) LV systolic dysfunction; and 3) metabolism of apoB-containing or triglyceride-rich lipoproteins.
\end{abstract}

Keywords: sphingomyelin left ventricular ejection fraction, coronary heart disease, lipids

\section{Introduction}

Sphingomyelin (SM), the second most abundant phospholipid in mammalian plasma, is present in all major lipoproteins. Up to $18 \%$ of total plasma phospholipid exists as SM [1], with the ratio of phosphatidylcholine (PC)/SM varying widely among lipoprotein subclasses [2]. Atherogenic lipoproteins such as VLDL and LDL are SM-enriched $[1,3]$. The SM content of atherosclerotic lesions is higher than that of normal arterial tissue [4].

Williams and Tabas have suggested that subendothelial retention and aggregation of atherogenic lipoproteins play an important role in atherogenesis $[5,6]$. SM-rich LDL retained in atherosclerotic lesions is acted on by an arterial wall sphingomyelinase that appears to promote aggregation, initiating the early phase of atherosclerosis development $[7,8]$. We have previously found that plasma SM levels in ApoE KO mice are 4-fold higher than those in WT mice [9], and this may partially explain the increased

\footnotetext{
* Correspondence: jbge@zshospital.net; jason.lazar@downstate.edu

+ Contributed equally

${ }^{1}$ Institute of Cardiology, Zhongshan Hospital, Fudan University, Shanghai, PR China

${ }^{2}$ Division of Cardiovascular Medicine, SUNY Downstate Medical Center, Brooklyn, NY, USA

Full list of author information is available at the end of the article
}

atherosclerosis found in these animals [10]. Our laboratory and others have also discovered that inhibition of SM biosynthesis significantly decreases plasma SM levels, thus lessening atherosclerotic lesions in ApoE KO mice [11,12].

In case-control studies by Jiang et al [13] and Schlitt et al [14], subjects with coronary heart disease (CHD) had higher plasma SM levels than control subjects. In the multi-ethnic study including 6814 subjects without clinical CHD at baseline by Nelson et al, more extensive subclinical atherosclerosis (carotid intimal-medial wall thickness, ankle-arm blood pressure index, and Agatston coronary artery calcium score) was associated with high plasma SM levels[15]. However, in the same population, Yeboah et al found plasma SM levels to be not predictive of incident CHD events after 5 years of follow-up [16]. Therefore, the question as to whether plasma SM is risk factor for CHD remains controversial. In this study, we investigated potential associations between SM and the presence of angiographic coronary heart disease (CHD) in a Chinese cohort.

\section{Methods}

Study Population: A total of 732 subjects who underwent diagnostic coronary angiography for chest pain in Zhongshan Hospital between January, 2004 and December, 
2005 were included in this study. Cardiovascular risk factors/diseases were obtained by clinical history. Coronary heart disease $(\mathrm{CHD})(\mathrm{n}=489)$ was predefined as the presence of a luminal diameter stenosis $\geq 50 \%$ in at least 1 major coronary artery territory (left anterior descending, left circumflex or right coronary artery or their major branches). Among CHD patients, 388 had stable angina pectoris (stable AP) and 101 had acute coronary syndrome (ACS). Non-CHD included subjects who had $<50 \%$ stenosis in all 3 coronary artery territories $(\mathrm{n}=$ 243). Exclusion criteria were evidence of significant concomitant cardiac and non-cardiac disease including severe valvular heart disease, prior known cardiomyopathy, malignancy, or febrile condition. Diabetes mellitus was diagnosed by clinical history, use of hypoglycemic medications or a fasting blood sugar level $>7.0 \mathrm{mmol} / \mathrm{L}$ (125 mg/dL); hypertension was defined in patients receiving antihypertensive treatment or with known diagnosis of hypertension (blood pressure $\geq 140 / 90 \mathrm{mmHg}$ ). The study was approved by the Institutional Review Board of Zhongshan Hospital, Fudan University.

Laboratory Evaluation: The laboratory evaluation was performed as described previously (17). Subjects were instructed to fast for at least 8 hours prior to blood sampling. Lipid profile was determined with Hitachi 7600 biochemistry autoanalyzer. Triglyceride (TG), total cholesterol (TC) and high-density lipoprotein-cholesterol (HDL-C) were measured with enzymatic methods (TG, Shanghai Kehuadongling Diagnostics Co, Ltd; TC, Shanghai Kehuadongling Diagnostics Co, Ltd; HDL-C, PEG-modified enzyme HDL-C assay, Kyowa Medex Co). Low-density lipoprotein-cholesterol (LDL-C) was calculated according to the Friedewald formula. ApoA-I, apoE, apoB, and Lipoprotein(a) $[\mathrm{Lp}(\mathrm{a})]$ were determined by immunoturbidimetric assays (apoA-I, apoE, and apoB, DiaSyA Diagnostics; Lp(a), Nittobo Boseki Co Ltd).

Plasma SM measurement was performed as described previously[17]. There were 4 steps to the enzymatic measurement of plasma SM levels: 1) bacterial SMase hydrolyzed SM to phosphorylcholine and n-acylsphingosine; 2) alkaline phosphatase generated choline from phosphorylcholine; 3 ) choline was then used to generate hydrogen peroxide in a reaction catalyzed by choline oxidase; and 4) lastly, hydrogen peroxide was used together with DAOS, 4-aminoantipyrine, and peroxidase, as a catalyst, to generate a blue dye with an optimal absorption at 595 $\mathrm{nm}$. The reaction buffer consisted of $0.05 \mathrm{M}$ Tris- $\mathrm{HCl}$ with $0.66 \mathrm{mM}$ calcium chloride, $\mathrm{pH}$ 8. The following enzyme concentrations present in a $50 \mathrm{ml}$ reaction buffer: 25 units of SMase, 500 units of alkaline phosphatase, 25 units of choline oxidase, and 1,000 units of peroxidase. DAOS concentration was $0.73 \mathrm{mM}$, and 4-aminoantipyrine concentration was $0.73 \mathrm{mM}$. Five microliters of plasma was added to a $100 \mu \mathrm{l}$ reaction buffer containing enzymes, and after $45 \mathrm{~min}$ of incubation at $37^{\circ} \mathrm{C}$, the absorption was measured at $595 \mathrm{~nm}$ on a spectrophotometric plate reader. The developed color remained constant after the incubation time. Standard SM solution (50 $\mathrm{mg} / \mathrm{dl}$ ) preparation (5 $\mathrm{mg}$ of SM) was dissolved in $10 \mathrm{ml}$ of $2 \%$ Triton X-100 ethanol solution. Each sample was measured in triplicate and the mean value was presented.

\section{Statistical Methods}

Continuous variables were reported as median and $25-75^{\text {th }}$ interquartile ranges if they were not normally distributed. The distributions for plasma concentrations of SM were skewed $(\mathrm{p}<0.001)$ on both the Kolmogorov-Smirnova and Shapiro-Wilks tests. Accordingly, SM levels are reported as median and $25-75^{\text {th }}$ percentiles. For univariate analyses, Spearman's correlation was used to determine correlations of SM with various atherosclerotic markers. SM levels were also compared among the CHD and nonCHD groups, among patients with and without diabetes, and among the normal LV function and LV dysfunction groups using the non-parametric Kruskal Wallis test. Forced logistic regression was used to determine the relation between SM levels and the presence of CHD using CHD as the dependent variable and SM levels, age, gender, BMI, left ventricular ejection fraction (LVEF), diabetes, smoking, HDL, TC, LDL, TG and hypertension as independent variables. The same independent variables were entered into a forced logistic model to identify predictors of LV systolic dysfunction. In addition, linear regression multivariate analysis was used to analyze predictors of LVEF as a continuous variable. Selected interaction effects were tested between the independent variables in the model. All analyses were done using SPSS version 17 analytical software (SPSS Inc., Chicago, IL).

\section{Results}

A total of 732 subjects were included in the study, 489 had CHD. Among the patients with CHD, 101 patients had ACS and 388 had stable AP. Clinical characteristics of the subjects are described in Table 1. Median SM levels were higher among patients with CHD than nonCHD patients (47.2 vs $40.7 \mathrm{mg} / \mathrm{dl}, \mathrm{p}<0.001)$ (Figure $1 \mathrm{~A}$ ).

Of the total 732 patients included in the study, 619 had assessment of LV systolic function. Among those with LVEF, $86.1 \%$ had normal LVEF (>50\%), 8.6\% had mild LV dysfunction (EF ranging between 40-49\%), 3.4\% had moderate LV dysfunction (EF ranging between 30-39\%), and $1.9 \%$ had marked LV dysfunction ( $E F<30 \%)$. The moderate and severe groups were grouped together because of the small numbers of patients. Median SM levels were higher among patients with LV systolic dysfunction $(\mathrm{LVEF}<50 \%)$ than in those with normal systolic function $(\mathrm{LVEF} \geq 50 \%)$ (48.5 vs. $45.5 \mathrm{mg} / \mathrm{dl}, \mathrm{p}=0.001)$. As 
Table 1 Group comparisons between Non CHD and CHD as median (25-75 percentiles IQR) or percentage

\begin{tabular}{llll}
\hline Variable & Non CHD & CHD & P value \\
\hline Sphingomyelin(mg/dl) & $40.7(33-50)$ & $47.2(39-58)$ & $<0.001$ \\
Age (years) & $59(53-67)$ & $64(57-71)$ & $<0.001$ \\
BMI & $24.7(22-26)$ & $24.4(23-26)$ & 1.0 \\
LVEF (\%) & $69(64-73)$ & $63(55-70)$ & $<0.001$ \\
Fibrinogen (mg/dl) & $248(212-307)$ & $294(236-356)$ & $<0.001$ \\
HDL-C (mmol/L) & $1.1(0.9-1.2)$ & $1.0(0.8-1.2)$ & $<0.001$ \\
LDL-C (mmol/L) & $2.5(2.0-3.0)$ & $2.6(2.1-3.0)$ & 0.37 \\
TC (mmol/L) & $4.3(3.7-5.0)$ & $4.3(3.8-4.9)$ & 0.35 \\
TG (mmole/L) & $1.5(1.1-2.0)$ & $1.5(1.1-2.2)$ & 0.60 \\
ApoA-I (g/L) & $1.1(1.0-1.2)$ & $1.1(0.9-1.2)$ & 0.03 \\
ApoB (g/L) & $0.7(0.6-0.9)$ & $0.8(0.6-0.9)$ & \\
\hline
\end{tabular}

CHD, coronary heart disease; BMI, body mass index; LVEF, left ventricular ejection fraction; HDL, high density lipoprotein; LDL, low density lipoprotein; TC, total cholesterol; TG, triglyceride; Apo, apolipoprotein.

shown in Figure 1B, there was a step-wise increase in median SM levels in patients with normal, mild, and moderate-severe degrees of LV dysfunction $(45.5 \mathrm{mg} / \mathrm{dL}$ vs. $47.3 \mathrm{mg} / \mathrm{dL}$ vs. $57.6 \mathrm{mg} / \mathrm{dl}, \mathrm{p}=0.021)$. When considered as a continuous variable, LVEF was significantly correlated with SM levels in all patients $(r=-0.20, p<$ $0.001)$, in those with CHD $(r=-0.15, p=0.003)$, and in those without $\mathrm{CHD}(\mathrm{r}=-0.14, \mathrm{p}=0.046)$.

On univariate analyses, SM levels were significantly correlated with triglyceride levels $(r=0.10, p=0.005)$, $\operatorname{apoB}(\mathrm{r}=0.13, \mathrm{p}=0.001)$, apoE $(\mathrm{r}=0.135, \mathrm{p}<0.001)$, fibrinogen levels $(\mathrm{r}=0.11, \mathrm{p}=0.006)$, and LVEF $(\mathrm{r}=$ $-0.201, p<0.001)($ Table 2$)$. There were no significant correlations between SM levels and HDL cholesterol $(\mathrm{r}=-0.069, \mathrm{p}=0.061)$, total cholesterol $(\mathrm{r}=0.064, \mathrm{p}=$ $0.089)$, or LDL cholesterol levels $(r=0.035, p=0.35)$. Median SM levels were higher for patients with diabetes as compared to those without ( 47.1 vs. $45.2, \mathrm{p}=0.029$ ).

Using multivariate logistic regression analysis including age, gender, BMI, smoking, hypertension, diabetes, HDL, LDL, TG, LV systolic dysfunction $(\mathrm{LVEF}<50 \%)$ and SM levels, variables that were independently associated with the presence of CHD were: age $(\mathrm{p}<0.0001)$, male gender $(\mathrm{p}<0.0001)$, smoking $(\mathrm{p}=0.002)$, diabetes $(\mathrm{p}=0.004)$, hypertension $(\mathrm{p}=0.03), \operatorname{LVEF}(\mathrm{p}<0.001)$ and SM $(\mathrm{p}<$ 0.001) (Table 3).

Using multivariate linear regression including the same variables to predict LVEF as a continuous variable,

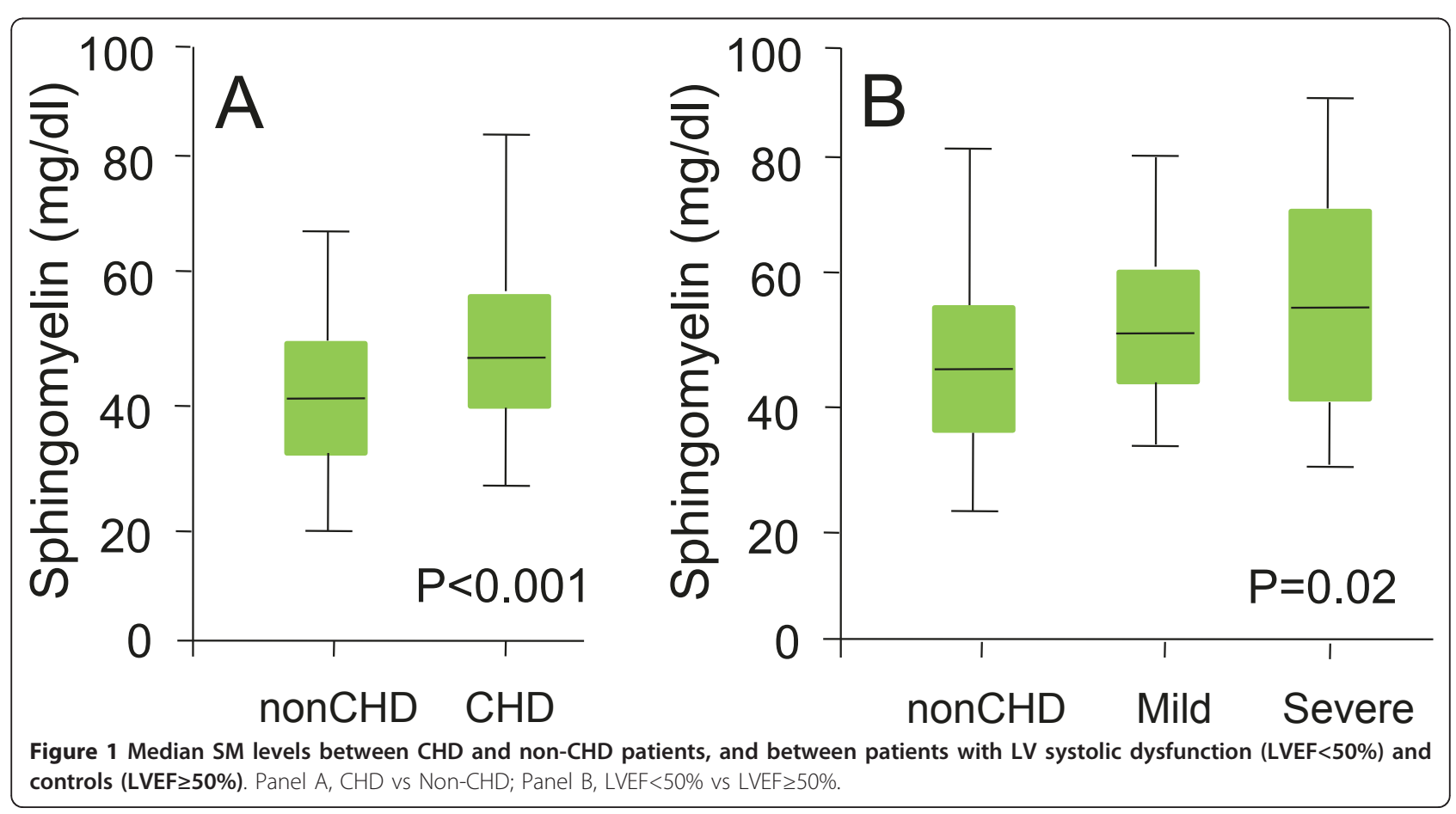


Table 2 Univariate correlations of sphingomyelin

\begin{tabular}{|c|c|c|}
\hline Variable & & Sphingomyelin (mg/dl) \\
\hline \multirow[t]{2}{*}{ Fibrinogen } & $r$ & 0.107 \\
\hline & $\mathrm{P}$ & 0.006 \\
\hline \multirow[t]{2}{*}{ LVEF } & $r$ & -0.201 \\
\hline & $\mathrm{P}$ & $<0.001$ \\
\hline \multirow[t]{2}{*}{ Аров } & r & 0.126 \\
\hline & $\mathrm{P}$ & $<0.001$ \\
\hline \multirow[t]{2}{*}{ ApoE } & $r$ & 0.135 \\
\hline & $\mathrm{P}$ & $<0.001$ \\
\hline \multirow[t]{2}{*}{$\mathrm{TG}$} & $r$ & 0.104 \\
\hline & $\mathrm{P}$ & 0.005 \\
\hline
\end{tabular}

LVEF, left ventricular ejection fraction; TG, triglyceride; Apo, apolipoprotein.

LVEF was independently related to SM levels ( $\mathrm{p}=$ 0.035 ) and a trend towards the presence of CHD ( $\mathrm{p}=$ 0.052) (Table 4).

\section{Discussion}

In the present study, we found that SM levels were independently associated with $\mathrm{CHD}$, which was in line with our previous observation [13]. Furthermore, we reveal that plasma SM levels were correlated with LV systolic dysfunction, and with plasma apoB and triglycerides levels.

It is known that atherosclerosis is an inflammatory disease. Inflammatory cells such as macrophages and lymphocytes and inflammatory factors including cytokines, chemokines and growth factors, promote focal necrosis and advanced complicated lesion formation. SM might act as a marker for an inflammatory effect in the development and progression of CHD. Our sphingomyelin syntase (SMS) 2 mouse studies indicated that $\mathrm{SM}$ biosynthesis is responsible for $\mathrm{NF}-\kappa \mathrm{B}$ and MAP kinase-mediated inflammatory and atherosclerosis

Table 3 Logistic regression predictors of CHD

\begin{tabular}{llll}
\hline Variable & P value & aOR & $95 \% \mathrm{CI}$ \\
\hline Sphingomyelin(mg/dl) & $<0.001$ & 1.056 & $(1.04-1.08)$ \\
Age (years) & $<0.0001$ & 1.062 & $(1.05-1.09)$ \\
Male gender & $<0.0001$ & 0.383 & $(.25-.63)$ \\
Smoking & 0.002 & 2.028 & $(1.3-2.99)$ \\
DM (history) & 0.004 & 2.292 & $(1.66-4.78)$ \\
HTN (history) & 0.03 & 1.596 & $(1.05-2.44)$ \\
HDL (mmol/L) & 0.071 & 0.445 & $(.202-1.10)$ \\
TG (mmol/L) & 0.085 & 0.981 & $(0.90-1.04)$ \\
LDL (mmol/L) & 0.084 & 1.210 & $(.98-1.50)$ \\
LVEF (\%) & $<0.001$ & 0.033 & $(.005-.212)$ \\
\hline
\end{tabular}

CHD, coronary heart disease; DM, diabetes; HTN,

hypertension; TG, triglyceride; HDL, high density

lipoprotein; LDL, low density lipoprotein; LVEF, left ventricular ejection fraction.
Table 4 Logistic regression predictors of LV systolic dysfunction

\begin{tabular}{llll}
\hline Variable & P value & aOR & 95\%CI \\
\hline Sphingomyelin(mg/dl) & 0.035 & 1.02 & $(1.00-1.03)$ \\
Age (years) & 0.885 & 0.99 & $(0.97-1.02)$ \\
Male gender & 0.074 & 0.534 & $(.27-1.06)$ \\
Smoking & 0.143 & 0.684 & $(.41-1.13)$ \\
DM (history) & 0.618 & 1.160 & $(.65-2.07)$ \\
HTN (history) & 0.309 & 0.772 & $(.47-1.27)$ \\
BMI & 0.107 & 1.851 & $(.99-3.44)$ \\
CHD & 0.052 & 0.540 & $(.29-1.00)$ \\
\hline
\end{tabular}

DM, diabetes; HTN, hypertension; BMI, body mass index; CHD, coronary heart disease.

$[18,19]$. We found that SMS2 deficiency substantially diminished the abundance of toll like receptor 4 (TLR4)-MD2 complex levels on the surface of macrophages after LPS stimulation [18]. Moreover, we found that SMS2 KO mice had less IL-6 and TNFa in the circulation after LPS stimulation, compared with controls [19].

The association between SM levels and LV dysfunction is a novel finding as we are unaware of prior studies reporting such a relation. Although CHD is a leading cause of LV systolic dysfunction, it is unlikely that the SM-LVEF relation is mediated by CHD. SM levels were similarly correlated in an inverse manner with LVEF in both patients with and without CHD. In addition, the relation between SM and LVEF remained significant despite inclusion of CHD in the multivariate model. It remains unclear as to whether higher plasma SM level is a marker of LV dysfunction or whether SM is directly involved in the pathogenesis of LV dysfunction. Alternatively, higher SM levels may represent an adaptive response to LV dysfunction. Inflammation is one of the possible linkages. In our patient population, there was a significant association between SM levels and fibrinogen, an acute phase reactant in inflammation. Prior studies have also found SM metabolism related to levels of CRP [20], inflammatory marker. Inflammation is well known to play a central role in the progression of LV dysfunction and heart failure [21]. Several lines of evidence suggest that SM may play a role in the development of LV dysfunction through promoting inflammation. In cardiomyocytes, activation of the neutral sphingomyelinase, which can hydrolyse SM, mediates TNF $\alpha$-induced apoptosis and negative contractile effect[22-25]. Sphingomyelinase levels have been shown to correlate with the presence and severity of congestive heart failure [26]. Moreover, Mrnka et al. showed that SM concentration in rat myocytes increased in response to pressure overload, a well known precursor to LV hypertrophy and dysfunction [27]. We previously showed that sphingomyelin synthase 2 (SMS 2), an enzyme directly involving in SM 
de novo biosynthesis, deficiency reduces plasma and cell membrane SM levels, reduces NF $\kappa \mathrm{B}-$, MAP kinasemediated inflammatory responses, and thus reduces atherosclerosis in mouse models[28,29]. However, there are few prior data establishing the direct relationship between plasma SM levels and LV dysfunction. This study is the first one. It is quite possible that SM levels, either in the blood or on the plasma membrane of cardio-muscle cells, play an important role in mediating LV dysfunction. The detail mechanism is deserved for further investigations.

In this study, we found the significant correlations between plasma SM and apoB or triglycerides (Table 2). It is well known that SM content is much higher in apoB-containing or triglyceride-rich lipoproteins than in HDL [1]. This may indicate that apoB-containing or triglyceride-rich lipoproteins are atherogenic but that HDL is not. It is also known that non-HDL lipoprotein subendothelial retention is an early step in atherogenesis [30]. It is believed that SM-rich non-HDL lipoproteins retained in atherosclerotic lesions are hydrolyzed by an arterial wall sphingomyelinase that promotes aggregation by converting SM to ceramide [5,31]. We have shown that plasma SM levels can serve as a marker for postprandial lipoprotein particle clearance [32]. If somehow this clearance was blocked, the SM-rich particles could likely be aggregated in the arterial wall after encountering sphingomylinase there, and development or instability of atheroscerotic plaques could be the consequence. Tabas's group provided convincing evidence that apoE KO mice lacking sphingomyelinase have decreased development of early atherosclerotic lesions [8]. We investigated this retention/aggregation event in another angle: reducing SM content of non-HDL lipoproteins through SMS deficient approach, thus leading to less non-HDL lipoprotein retention/aggregation in aorta, and preventing the development of atherosclerosis [33].

This study has a number of limitations. First, the independent relation of SM levels with LV systolic dysfunction should ideally be studied in the context of more established biomarkers of LV systolic dysfunction, such as BNP. Second, given the relatively small number of patients with ACS $(n=101)$ in our study population, we were unable to perform meaningful subgroup analyses of the relation between SM levels and ACS. Third, the size of the population was relatively small and the study was not designed with a priori calculations with respect to sample size or statistical power. As such, the findings need to be confirmed in larger and prospectively designed studies. Fourth, there is a difference in the age of the two cohorts that were compared. The observed changes in SM may reflect aging-associated differences. Lastly, we used the definition of coronary stenoses as defined $>50 \%$ by coronary angiography as a crude marker of hemodyanamically significant disease. Therefore, the control group likely did have atherosclerosis. Although numerous studies have used this criterion, quantification of coronary atherosclerosis by intravascular ultrasound is a more sensitive measure of atherosclerotic burden. However, this technique was not performed in the cohort evaluated in the present study.

In conclusion, in this study we found that the proatherogenic property of plasma SM might be related to 1) CHD; 2) LV systolic dysfunction; and 3) abnormal metabolism of apoB-containing or triglyceride-rich lipoproteins.

\section{Consent}

Written informed consent was obtained from the patient for publication of this case report and accompanying images. A copy of the written consent is available for review by the Editor-in-Chief of this journal.

\section{Acknowledgements}

This work was partially supported by National Institutes of Health grant HL093419 and HL093419-01A1; National Basic Research Program of China [2011CB503905], 863 Program of Science and Technology Ministry [2006AA0ZA406], Outstanding Youth Grant from National Natural Science Foundation of China [30725036].

\section{Author details}

${ }^{1}$ Institute of Cardiology, Zhongshan Hospital, Fudan University, Shanghai, PR China. ${ }^{2}$ Division of Cardiovascular Medicine, SUNY Downstate Medical Center, Brooklyn, NY, USA. ${ }^{3}$ Department of Cell Biology, SUNY Downstate Medical Center, Brooklyn, NY, USA.

\section{Authors' contributions}

$X C$ and AS carried out the SM measurement and modified the manuscript. $Y Z$ and JG provided the samples. JML participated in the design of the study, performed the statistical analysis, and partially drafted the manuscript. XCJ conceived of the study, participated in its design and coordination, and drafted the manuscript. All authors read and approved the final manuscript.

\section{Competing interests}

The authors declare that they have no competing interests.

Received: 30 January 2011 Accepted: 26 April 2011

Published: 26 April 2011

\section{References}

1. Nilsson A, Duan RD: Absorption and lipoprotein transport of sphingomyelin. J Lipid Res 2006, 47:154-171.

2. Subbaiah PV, Davidson MH, Ritter MC, Buchanan W, Bagdade JD: Effects of dietary supplementation with marine lipid concentrate on the plasma lipoprotein composition of hypercholesterolemic patients. Atherosclerosis 1989, 79:157-166.

3. Rodriguez JL, Ghiselli GC, Torreggiani D, Sirtori CR: Very low density lipoproteins in normal and cholesterol-fed rabbits: lipid and protein composition and metabolism. Part 1. Chemical composition of very low density lipoproteins in rabbits. Atherosclerosis 1976, 23:73-83.

4. Zilversmit DB, Mc CE, Jordan PH, Henly WS, Ackerman RF: The synthesis of phospholipids in human atheromatous lesions. Circulation 1961, 23:370-375.

5. Williams KJ, Tabas I: The response-to-retention hypothesis of early atherogenesis. Arterioscler Thromb Vasc Biol 1995, 15:551-561.

6. Williams KJ, Tabas I: The response-to-retention hypothesis of atherogenesis reinforced. Curr Opin Lipidol 1998, 9:471-474. 
7. Schissel SL, Tweedie-Hardman J, Rapp JH, Graham G, Williams KJ, Tabas I: Rabbit aorta and human atherosclerotic lesions hydrolyze the sphingomyelin of retained low-density lipoprotein. Proposed role for arterial-wall sphingomyelinase in subendothelial retention and aggregation of atherogenic lipoproteins. J Clin Invest 1996, 98:1455-1464.

8. Devlin CM, Leventhal AR, Kuriakose G, Schuchman EH, Williams KJ, Tabas I: Acid sphingomyelinase promotes lipoprotein retention within early atheromata and accelerates lesion progression. Arterioscler Thromb Vasc Biol 2008, 28:1723-1730.

9. Jeong T, Schissel SL, Tabas I, Pownall HJ, Tall AR, Jiang X: Increased sphingomyelin content of plasma lipoproteins in apolipoprotein $\mathrm{E}$ knockout mice reflects combined production and catabolic defects and enhances reactivity with mammalian sphingomyelinase. J Clin Invest 1998, 101:905-912

10. Plump AS, Smith JD, Hayek T, Aalto-Setala K, Walsh A, Verstuyft JG, Rubin EM, Breslow JL: Severe hypercholesterolemia and atherosclerosis in apolipoprotein E-deficient mice created by homologous recombination in ES cells. Cell 1992, 71:343-353.

11. Hojjati MR, Li Z, Zhou H, Tang S, Huan C, Ooi E, Lu S, Jiang XC: Effect of myriocin on plasma sphingolipid metabolism and atherosclerosis in apoE-deficient mice. J Biol Chem 2005, 280:10284-10289.

12. Park TS, Panek RL, Mueller SB, Hanselman JC, Rosebury WS, Robertson AW, Kindt EK, Homan R, Karathanasis SK, Rekhter MD: Inhibition of sphingomyelin synthesis reduces atherogenesis in apolipoprotein Eknockout mice. Circulation 2004, 110:3465-3471.

13. Jiang XC, Paultre F, Pearson TA, Reed RG, Francis CK, Lin M, Berglund L, Tall AR: Plasma sphingomyelin level as a risk factor for coronary artery disease. Arterioscler Thromb Vasc Biol 2000, 20:2614-2618.

14. Schlitt A, Blankenberg S, Yan D, von Gizycki H, Buerke M, Werdan K, Bickel C, Lackner KJ, Meyer J, Rupprecht HJ, Jiang XC: Further evaluation of plasma sphingomyelin levels as a risk factor for coronary artery disease. Nutr Metab (Lond) 2006, 3:5.

15. Nelson JC, Jiang XC, Tabas I, Tall A, Shea S: Plasma sphingomyelin and subclinical atherosclerosis: findings from the multi-ethnic study of atherosclerosis. Am J Epidemiol 2006, 163:903-912.

16. Yeboah J, McNamara C, Jiang XC, Tabas I, Herrington DM, Burke GL, Shea S: Association of plasma sphingomyelin levels and incident coronary heart disease events in an adult population: Multi-Ethnic Study of Atherosclerosis. Arterioscler Thromb Vasc Biol 2010, 30:628-633.

17. Hojjati MR, Jiang XC: Rapid, specific, and sensitive measurements of plasma sphingomyelin and phosphatidylcholine. J Lipid Res 2006, 47:673-676.

18. Hailemariam TK, Huan C, Liu J, Li Z, Roman C, Kalbfeisch M, Bui HH, Peake DA, Kuo MS, Cao G, Wadgaonkar R, Jiang XC: Sphingomyelin synthase 2 deficiency attenuates NFkappaB activation. Arterioscler Thromb Vasc Biol 2008, 28:1519-1526.

19. Liu JC, Wang DM, Qian JN, Li YG, Wang LJ, Jiang XL, Zhai LL, Lu J, Qi P: [Changes of nitric oxide and endothelin serum level after carotid balloon denudation or stent assisted angioplasty: an experimental and clinical observation]. Zhonghua Wai Ke Za Zhi 2009, 47:423-426.

20. Lozanski G, Berthier F, Kushner I: The sphingomyelin-ceramide pathway participates in cytokine regulation of $\mathrm{C}$-reactive protein and serum amyloid A, but not alpha-fibrinogen. Biochem J 1997, 328(Pt 1):271-275.

21. Redfield MM, Jacobsen SJ, Burnett JC Jr, Mahoney DW, Bailey KR, Rodeheffer RJ: Burden of systolic and diastolic ventricular dysfunction in the community: appreciating the scope of the heart failure epidemic. JAMA 2003, 289:194-202.

22. Liu B, Andrieu-Abadie N, Levade T, Zhang P, Obeid LM, Hannun YA: Glutathione regulation of neutral sphingomyelinase in tumor necrosis factor-alpha-induced cell death. J Biol Chem 1998, 273:11313-11320.

23. Oral H, Dorn GW, Mann DL: Sphingosine mediates the immediate negative inotropic effects of tumor necrosis factor-alpha in the adult mammalian cardiac myocyte. J Biol Chem 1997, 272:4836-4842.

24. Amadou A, Nawrocki A, Best-Belpomme M, Pavoine C, Pecker F: Arachidonic acid mediates dual effect of TNF-alpha on $\mathrm{Ca} 2+$ transients and contraction of adult rat cardiomyocytes. Am J Physiol Cell Physiol 2002, 282:C1339-1347

25. Cailleret M, Amadou A, Andrieu-Abadie N, Nawrocki A, Adamy C, AitMamar B, Rocaries F, Best-Belpomme M, Levade T, Pavoine C, Pecker F: Nacetylcysteine prevents the deleterious effect of tumor necrosis factor- (alpha) on calcium transients and contraction in adult rat cardiomyocytes. Circulation 2004, 109:406-411.

26. Pavoine $C$, Pecker F: Sphingomyelinases: their regulation and roles in cardiovascular pathophysiology. Cardiovasc Res 2009, 82:175-183.

27. Mrnka L, Novakova O, Pelouch V, Novak F: Phospholipid composition in the rat heart exposed to pressure overload from birth. Physiol Res 1996, 45:83-85.

28. Liu J, Huan C, Chakraborty M, Zhang H, Lu D, Kuo MS, Cao G, Jiang XC: Macrophage sphingomyelin synthase 2 deficiency decreases atherosclerosis in mice. Circ Res 2009, 105:295-303.

29. Fan Y, Shi F, Liu J, Dong J, Bui HH, Peake DA, Kuo MS, Cao G, Jiang XC: Selective reduction in the sphingomyelin content of atherogenic lipoproteins inhibits their retention in murine aortas and the subsequent development of atherosclerosis. Arterioscler Thromb Vasc Biol 2010, 30:2114-2120.

30. Skalen K, Gustafsson M, Rydberg EK, Hulten LM, Wiklund O, Innerarity TL, Boren J: Subendothelial retention of atherogenic lipoproteins in early atherosclerosis. Nature 2002, 417:750-754.

31. Schissel SL, Keesler GA, Schuchman EH, Williams KJ, Tabas I: The cellular trafficking and zinc dependence of secretory and lysosomal sphingomyelinase, two products of the acid sphingomyelinase gene. J Biol Chem 1998, 273:18250-18259.

32. Schlitt A, Hojjati MR, von Gizycki H, Lackner KJ, Blankenberg S, Schwaab B, Meyer J, Rupprecht HJ, Jiang XC: Serum sphingomyelin levels are related to the clearance of postprandial remnantlike particles. J Lipid Res 2005, 46:196-200.

33. Fan Y, Shi F, Liu J, Dong J, Bui HH, Peake DA, Kuo MS, Cao G, Jiang XC: Selective Reduction in the Sphingomyelin Content of Atherogenic Lipoproteins Inhibits Their Retention in Murine Aortas and the Subsequent Development of Atherosclerosis. Arterioscler Thromb Vasc Biol 2010.

doi:10.1186/1743-7075-8-25

Cite this article as: Chen et al:: Impact of sphingomyelin levels on coronary heart disease and left ventricular systolic function in humans. Nutrition \& Metabolism 2011 8:25.

\section{Submit your next manuscript to BioMed Central and take full advantage of:}

- Convenient online submission

- Thorough peer review

- No space constraints or color figure charges

- Immediate publication on acceptance

- Inclusion in PubMed, CAS, Scopus and Google Scholar

- Research which is freely available for redistribution 